\title{
Updated MiniBooNE neutrino oscillation results with increased data and new background studies
}

A. A. Aguilar-Arevalo, ${ }^{14}$ B. C. Brown, ${ }^{5}$ J. M. Conrad,${ }^{13}$ R. Dharmapalan,,${ }^{1,7}$ A. Diaz, ${ }^{13}$ Z. Djurcic, ${ }^{2}$ D. A. Finley, ${ }^{5}$ R. Ford, ${ }^{5}$ G. T. Garvey, ${ }^{10}$ S. Gollapinni, ${ }^{10}$ A. Hourlier, ${ }^{13}$ E.-C. Huang, ${ }^{10}$ N. W. Kamp ${ }^{13}$ G. Karagiorgi, ${ }^{4}$ T. Katori, ${ }^{12}$ T. Kobilarcik, ${ }^{5}$ K. Lin, ${ }^{4,10}$ W. C. Louis $\odot,{ }^{10}$ C. Mariani,${ }^{17}$ W. Marsh, ${ }^{5}$ G. B. Mills, ${ }^{10,}$ J. Mirabal-Martinez, ${ }^{10}$ C. D. Moore, ${ }^{5}$ R. H. Nelson, ${ }^{3, \dagger}$ J. Nowak, ${ }^{9}$ I. Parmaksiz, ${ }^{16}$ Z. Pavlovic, ${ }^{5}$ H. Ray ${ }^{6}$ B. P. Roe, ${ }^{15}$ A. D. Russell, ${ }^{5}$ A. Schneider, ${ }^{13}$ M. H. Shaevitz, ${ }^{4}$ H. Siegel,${ }^{4}$ J. Spitz, ${ }^{15}$ I. Stancu, ${ }^{1}$ R. Tayloe, ${ }^{8}$ R. T. Thornton, ${ }^{10}$ M. Tzanov, ${ }^{3,11}$ R. G. Van de Water, ${ }^{10}$ D. H. White, ${ }^{10, *}$ and E. D. Zimmerman ${ }^{3}$

(MiniBooNE Collaboration)

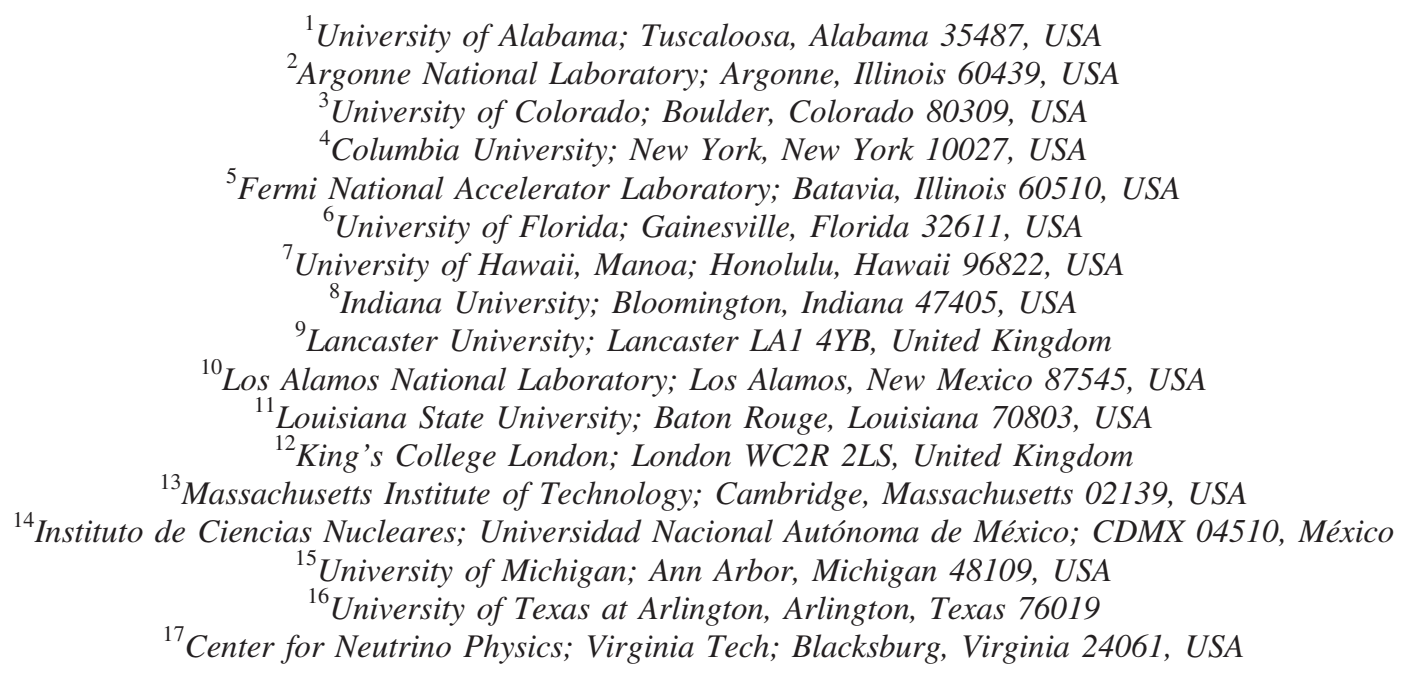

(Received 13 November 2020; accepted 22 February 2021; published 8 March 2021)

The MiniBooNE experiment at Fermilab reports a total excess of $638.0 \pm 52.1$ (stat.) \pm 122.2 (syst.) electronlike events from a data sample corresponding to $18.75 \times 10^{20}$ protons-on-target in neutrino mode, which is a $46 \%$ increase in the data sample with respect to previously published results and $11.27 \times 10^{20}$ protons-on-target in antineutrino mode. The overall significance of the excess, $4.8 \sigma$, is limited by systematic uncertainties, assumed to be Gaussian, as the statistical significance of the excess is $12.2 \sigma$. The additional statistics allow several studies to address questions on the source of the excess. First, we provide two-dimensional plots in visible energy and the cosine of the angle of the outgoing lepton, which can provide valuable input to models for the event excess. Second, we test whether the excess may arise from photons that enter the detector from external events or photons exiting the detector from $\pi^{0}$ decays in two model independent ways. Beam timing information shows that almost all of the excess is in time with neutrinos that interact in the detector. The radius distribution shows that the excess is distributed throughout the volume, while tighter cuts on the fiducial volume increase the significance of the excess. The data likelihood ratio disfavors models that explain the event excess due to entering or exiting photons.

DOI: $10.1103 /$ PhysRevD.103.052002

\footnotetext{
*Deceased.

Present address: The Aerospace Corporation, Los Angeles, California 90009, USA.

Published by the American Physical Society under the terms of the Creative Commons Attribution 4.0 International license. Further distribution of this work must maintain attribution to the author(s) and the published article's title, journal citation, and DOI. Funded by SCOAP.
}

\section{INTRODUCTION}

The LSND [1] and MiniBooNE [2,3] experiments have reported excesses of $\nu_{e}$ and $\bar{\nu}_{e}$ charge-current quasielastic (CCQE) events in $\nu_{\mu}$ beams. Exotic models beyond the three-neutrino paradigm that have been invoked to explain these anomalies include, for example, $3+\mathrm{N}$ neutrino 
oscillation models involving three active neutrinos and $\mathrm{N}$ additional sterile neutrinos [4-14], resonant neutrino oscillations [15], Lorentz violation [16], sterile neutrino decay [17], scalar decay [18], sterile neutrino nonstandard interactions [19], and altered dispersion relations with sterile neutrinos [20]. This paper presents improved MiniBooNE $\nu_{e}$ appearance results with increased statistics and with additional studies that disfavor neutral-current (NC) $\pi^{0}$ and external event backgrounds.

\section{THE MINIBOONE EXPERIMENT}

The MiniBooNE experiment makes use of the Booster Neutrino Beam (BNB) that is produced by $8 \mathrm{GeV}$ protons from the Fermilab Booster interacting on a beryllium target inside a magnetic focusing horn, followed by meson decay in a $50 \mathrm{~m}$ decay pipe. In neutrino mode, the $\nu_{\mu}, \bar{\nu}_{\mu}, \nu_{e}$, and $\bar{\nu}_{e}$ flux contributions at the detector are $93.5 \%, 5.9 \%, 0.5 \%$, and $0.1 \%$, respectively, while in antineutrino mode, the flux contributions are $15.7 \%$, $83.7 \%, 0.2 \%$, and $0.4 \%$, respectively. The $\nu_{\mu}$ and $\bar{\nu}_{\mu}$ fluxes peak at approximately $600 \mathrm{MeV}$ and $400 \mathrm{MeV}$, respectively. The MiniBooNE detector, described in detail in Ref. [21], consists of a $12.2 \mathrm{~m}$ diameter sphere filled with 818 tonnes of pure mineral oil $\left(\mathrm{CH}_{2}\right)$ and is located $541 \mathrm{~m}$ from the beryllium target. The detector is covered by 1520 8-inch photomultiplier tubes (PMTs), where 1280 PMTs are in the interior detector region and 240 PMTs are located in the optically isolated outer veto region. The PMTs detect the directed Cherenkov light and the isotropic scintillation light produced by charged particles from neutrino interactions in the mineral oil. Events are reconstructed [22] from the hit PMT charge and time information, and the reconstructed neutrino energy, $E_{\nu}^{\mathrm{QE}}$, is estimated from the measured energy and angle of the outgoing muon or electron, assuming the kinematics of CCQE scattering [23]. The MiniBooNE experiment has collected data from 2002-2019, based on a total of $11.27 \times 10^{20}$ protons-on-target (POT) in antineutrino mode and $18.75 \times 10^{20}$ POT in neutrino mode. Also, a special beam off-target run collected an additional $1.86 \times 10^{20}$ POT in a search for sub-GeV dark matter [24]. During the 17 years of running, the BNB and MiniBooNE detector have been stable to within 3\% in neutrino energy. Figure 1 shows the energy distribution of Michel electrons from stopped muon decay for the first $\left(6.46 \times 10^{20}\right.$ POT from 2002 to 2007), second $\left(6.38 \times 10^{20}\right.$ POT from 2015 to 2017$)$, and third running periods $\left(5.91 \times 10^{20}\right.$ POT from 2017 to 2019$)$ in neutrino mode. By adjusting the energy calibration by $2 \%$ for the second running period and by $3 \%$ for the third running period, good agreement is obtained for the Michel electron energy distribution.
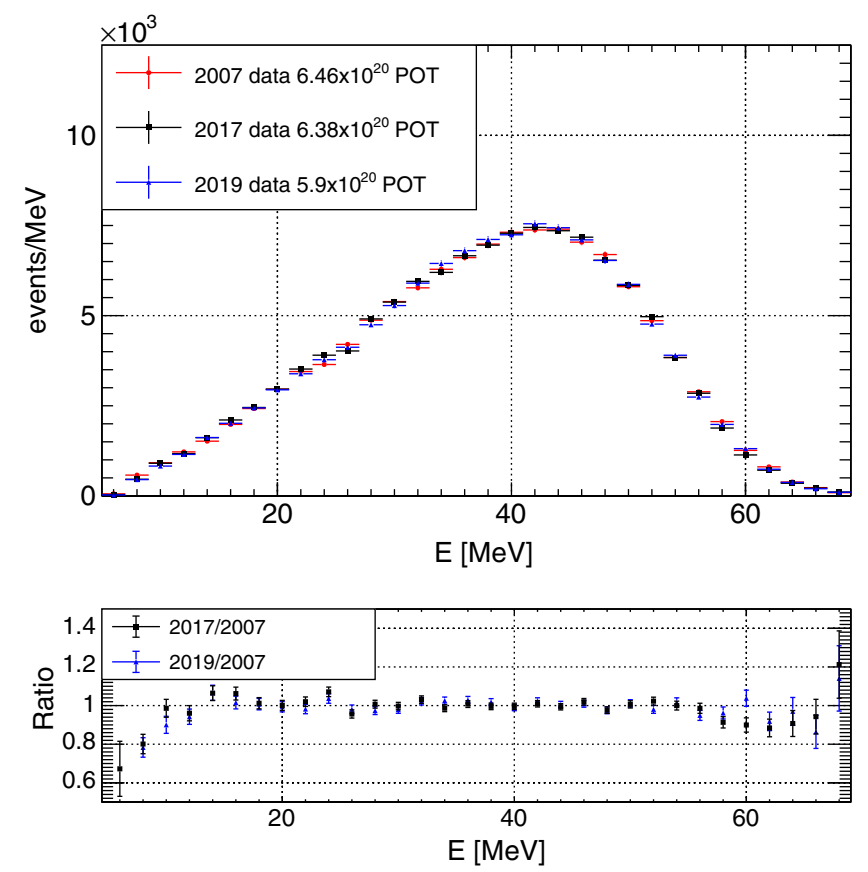

FIG. 1. The Michel electron energy distribution for the first, second, and third running periods in neutrino mode. The events are normalized to the first running period. The bottom plot shows ratios of the second and third running periods to the first running period.

\section{DATA ANALYSIS}

The data analysis is optimized to measure $\nu_{e}$-induced $\mathrm{CCQE}$ events and reject $\nu_{\mu}$ induced events, and is identical to the previous analysis [2]. Figures $2-4$ show the $\nu_{e} \mathrm{CCQE}$ data and background for the three particle identification

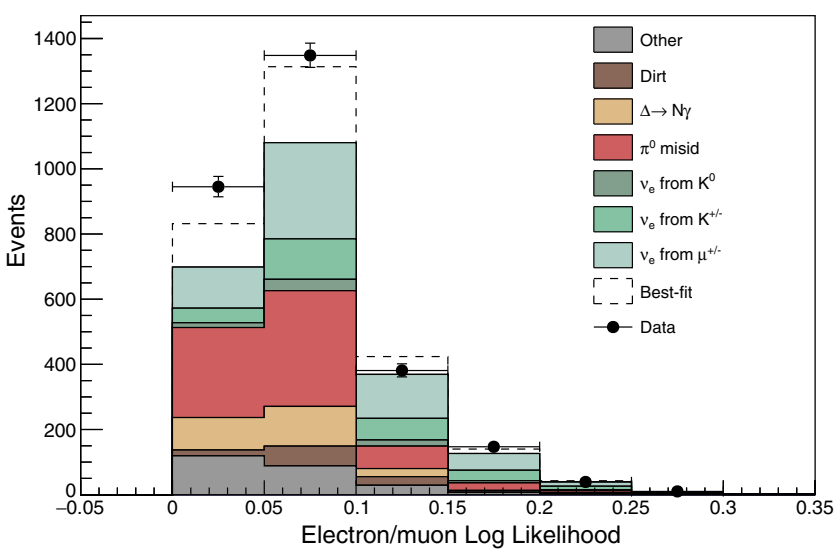

FIG. 2. The MiniBooNE neutrino mode electron-muon particle identification distributions, corresponding to the total $18.75 \times$ $10^{20}$ POT data in the $200<E_{\nu}^{\mathrm{QE}}<1250 \mathrm{MeV}$ energy range, for $\nu_{e}$ CCQE data (points with statistical errors) and background (colored histogram). The dashed histogram shows the best fit to the neutrino-mode data assuming two-neutrino oscillations. 


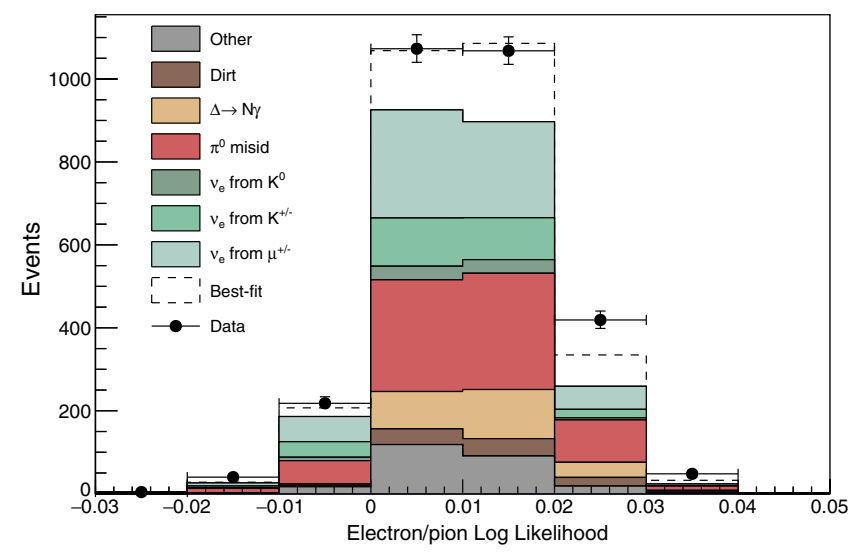

FIG. 3. The MiniBooNE neutrino mode electron-pion particle identification distributions, corresponding to the total $18.75 \times$ $10^{20}$ POT data in the $200<E_{\nu}^{\mathrm{QE}}<1250 \mathrm{MeV}$ energy range, for $\nu_{e}$ CCQE data (points with statistical errors) and background (colored histogram). The dashed histogram shows the best fit to the neutrino-mode data assuming two-neutrino oscillations.

variables in neutrino mode in the $200<E_{\nu}^{\mathrm{QE}}<1250 \mathrm{MeV}$ energy range for the total $18.75 \times 10^{20}$ POT data. The comparison between data and background for the full range of particle identification variables was shown in the Supplemental Material of a previous publication [3]. The average selection efficiency is $\sim 20 \%(\sim 0.1 \%)$ for $\nu_{e}$-induced CCQE events $\left(\nu_{\mu}\right.$-induced background events) generated over the fiducial volume. The fraction of CCQE events in antineutrino mode that are from wrongsign neutrino events was determined from the angular distributions of muons created in CCQE interactions and by measuring CC single $\pi^{+}$events [25]. Table I shows

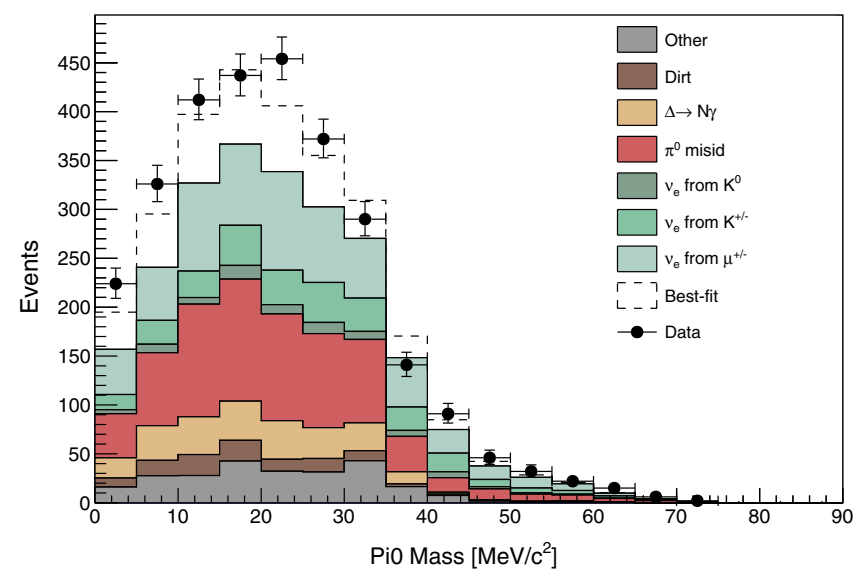

FIG. 4. The MiniBooNE neutrino mode two-ring invariantmass particle identification distributions, corresponding to the total $18.75 \times 10^{20}$ POT data in the $200<E_{\nu}^{\mathrm{QE}}<1250 \mathrm{MeV}$ energy range, for $\nu_{e} \mathrm{CCQE}$ data (points with statistical errors) and background (colored histogram). The dashed histogram shows the best fit to the neutrino-mode data assuming two-neutrino oscillations.
TABLE I. The expected (unconstrained) number of events for the $200<E_{\nu}^{\mathrm{QE}}<1250 \mathrm{MeV}$ neutrino energy range from all of the backgrounds in the $\nu_{e}$ and $\bar{\nu}_{e}$ appearance analysis before using the constraint from the $\mathrm{CC} \nu_{\mu}$ events. The "other" backgrounds correspond mostly to neutrino-nucleon and neutrinoelectron elastic scattering. Also shown are the constrained background, as well as the expected number of events corresponding to the LSND best fit oscillation probability of $0.26 \%$, assuming oscillations at large $\Delta m^{2}$. The table shows the diagonalelement systematic plus statistical uncertainties, which become substantially reduced in the oscillation fits when correlations between energy bins and between the $\nu_{e}$ and $\nu_{\mu}$ events are included.

\begin{tabular}{lcc}
\hline \hline Process & Neutrino mode & Antineutrino mode \\
\hline$\nu_{\mu}$ and $\bar{\nu}_{\mu} \mathrm{CCQE}$ & $107.6 \pm 28.2$ & $12.9 \pm 4.3$ \\
$\mathrm{NC} \pi^{0}$ & $732.3 \pm 95.5$ & $112.3 \pm 11.5$ \\
$\mathrm{NC} \Delta \rightarrow N \gamma$ & $251.9 \pm 35.2$ & $34.7 \pm 5.4$ \\
External events & $109.8 \pm 15.9$ & $15.3 \pm 2.8$ \\
Other $\nu_{\mu}$ and $\bar{\nu}_{\mu}$ & $130.8 \pm 33.4$ & $22.3 \pm 3.5$ \\
$\nu_{e}$ and $\bar{\nu}_{e}$ from $\mu^{ \pm}$decay & $621.1 \pm 146.3$ & $91.4 \pm 27.6$ \\
$\nu_{e}$ and $\bar{\nu}_{e}$ from $K^{ \pm}$decay & $280.7 \pm 61.2$ & $51.2 \pm 11.0$ \\
$\nu_{e}$ and $\bar{\nu}_{e}$ from $K_{L}^{0}$ decay & $79.6 \pm 29.9$ & $51.4 \pm 18.0$ \\
Other $\nu_{e}$ and $\bar{\nu}_{e}$ & $8.8 \pm 4.7$ & $6.7 \pm 6.0$ \\
Unconstrained bkgd. & $2322.6 \pm 258.3$ & $398.2 \pm 49.7$ \\
Constrained bkgd. & $2309.4 \pm 119.6$ & $400.6 \pm 28.5$ \\
Total data & 2870 & 478 \\
Excess & $560.6 \pm 119.6$ & $77.4 \pm 28.5$ \\
$0.26 \%$ (LSND) $\nu_{\mu} \rightarrow \nu_{e}$ & 676.3 & 100.0 \\
\hline \hline
\end{tabular}

the predicted but unconstrained $\nu_{e}$ and $\bar{\nu}_{e}$ CCQE background events for the neutrino energy range $200<E_{\nu}^{\mathrm{QE}}<$ $1250 \mathrm{MeV}$ for both neutrino and antineutrino modes, where there are approximately twice as many Monte Carlo events compared to data events. Table I also shows the total constrained background, where the overall normalization of the $\nu_{e}$ intrinsic background is constrained by the $\nu_{\mu}$ CCQE event sample. The upper limit of $1250 \mathrm{MeV}$ was chosen by the Collaboration before unblinding the data in 2007, while the lower limit of $200 \mathrm{MeV}$ was chosen in 2013 [2], because it is the lowest energy for reliably reconstructing the Cherenkov ring of $\nu_{\mu}$ CCQE events with a visible energy greater than $140 \mathrm{MeV}$. From the given detector resolution estimated from the Michel electron spectrum (Fig. 1), there is a negligible amount of migration from events below $200 \mathrm{MeV}$. Note that the original lower limit was chosen to be $300 \mathrm{MeV}$ before unblinding the data in 2007. During the unblinding procedure, the lower limit was increased to $475 \mathrm{MeV}$ due to the low probability of the two-neutrino oscillation fit and worries about the single-gamma background. However, careful studies of the single-gamma background were performed after unblinding and convinced the Collaboration that the single-gamma background was 

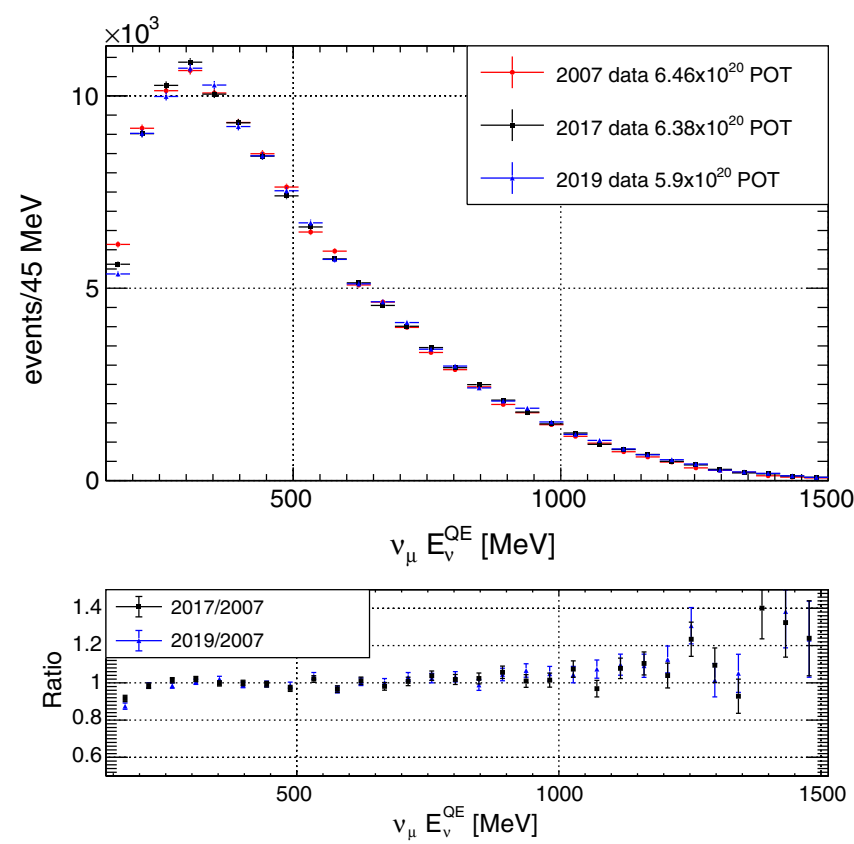

FIG. 5. The $\nu_{\mu}$ CCQE muon visible energy distribution for the first, second, and third running periods in neutrino mode. The events are normalized to the first running period. The bottom plot shows ratios of the second and third running periods to the first running period.

estimated correctly within systematic uncertainties and agreed with theoretical calculations [26]. Finally, Table I shows the expected number of events corresponding to the LSND best fit oscillation probability of $0.26 \%$, assuming
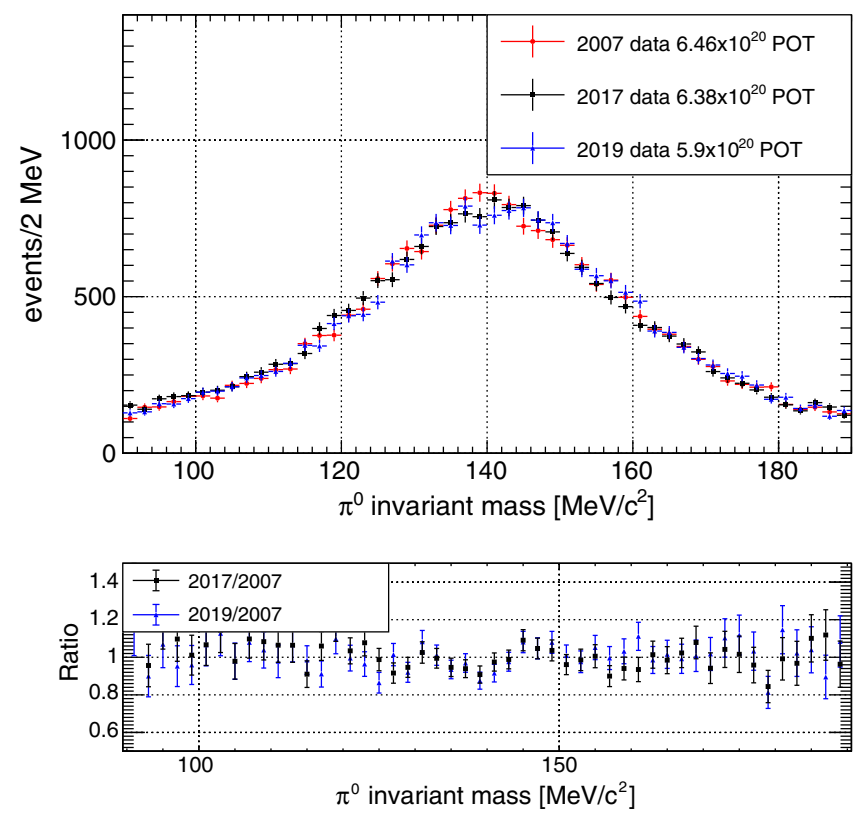

FIG. 6. The NC $\pi^{0}$ mass distribution for the first, second, and third running periods in neutrino mode. The events are normalized to the first running period. The bottom plot shows ratios of the second and third running periods to the first running period.

large $\Delta m^{2}$ where the oscillations are washed out. LSND and MiniBooNE have the same average value of $\mathrm{L} / \mathrm{E}$, but MiniBooNE has a larger range of $\mathrm{L} / \mathrm{E}$. Therefore, the appearance probabilities for LSND and MiniBooNE should not be exactly the same at lower $\mathrm{L} / \mathrm{E}$ values. Figures 5 and 6 show the $\nu_{\mu} \mathrm{CCQE} E_{\nu}^{\mathrm{QE}}$ energy distribution and the $\mathrm{NC} \pi^{0}$ mass distribution in neutrino mode for the first, second, and third running periods. As shown in the figures, the three running periods have good agreement.

Systematic uncertainties are determined by considering the predicted effects on the $\nu_{\mu}, \bar{\nu}_{\mu}, \nu_{e}$, and $\bar{\nu}_{e}$ CCQE rates from variations of model parameters that include uncertainties in the neutrino and antineutrino flux estimates, uncertainties in neutrino cross sections, uncertainties from nuclear effects, and uncertainties in detector modeling and reconstruction. A covariance matrix in bins of $E_{\nu}^{\mathrm{QE}}$ is constructed by considering the variation from each source of systematic uncertainty on the $\nu_{e}$ and $\bar{\nu}_{e} \mathrm{CCQE}$ signal and background, and the $\nu_{\mu}$ and $\bar{\nu}_{\mu}$ CCQE prediction as a function of $E_{\nu}^{\mathrm{QE}}$. This matrix includes correlations between any of the $\nu_{e}$ and $\bar{\nu}_{e} \mathrm{CCQE}$ signal and background and $\nu_{\mu}$ and $\bar{\nu}_{\mu} \mathrm{CCQE}$ samples, and is used in the $\chi^{2}$ calculation of the oscillation fits.

\section{ELECTRON-NEUTRINO APPEARANCE RESULTS}

Figures 7-9 show the visible energy, $\cos \theta$, and $E_{\nu}^{\mathrm{QE}}$ distributions for $\nu_{e} \mathrm{CCQE}$ data and background in neutrino mode in the $200<E_{\nu}^{\mathrm{QE}}<1250 \mathrm{MeV}$ energy range for the total $18.75 \times 10^{20}$ POT data, where $\theta$ is the angle of the reconstructed electron relative to the incident beam direction. Each bin of reconstructed $E_{\nu}^{\mathrm{QE}}$ corresponds to a

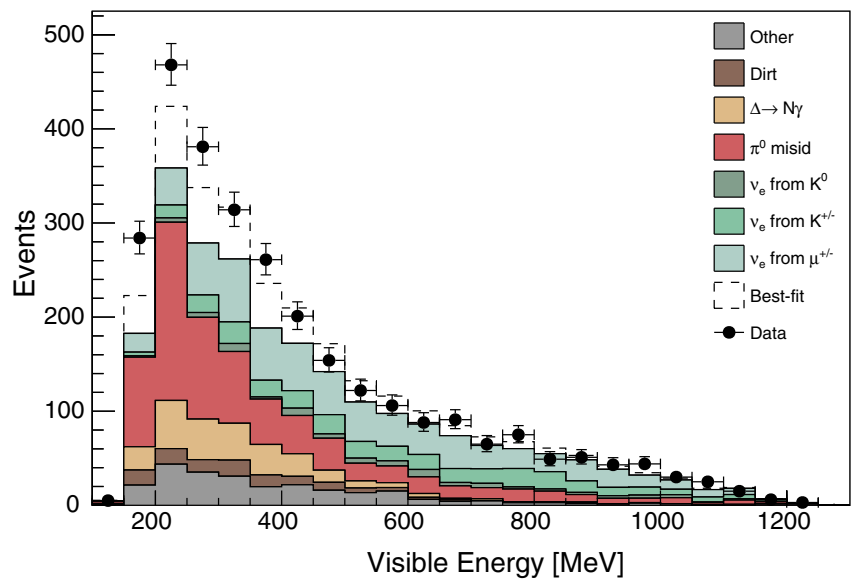

FIG. 7. The MiniBooNE neutrino mode visible energy distributions, corresponding to the total $18.75 \times 10^{20}$ POT data in the $200<E_{\nu}^{\mathrm{QE}}<1250 \mathrm{MeV}$ energy range, for $\nu_{e} \mathrm{CCQE}$ data (points with statistical errors) and background (colored histogram). The dashed histogram shows the best fit to the neutrino-mode data assuming two-neutrino oscillations. 


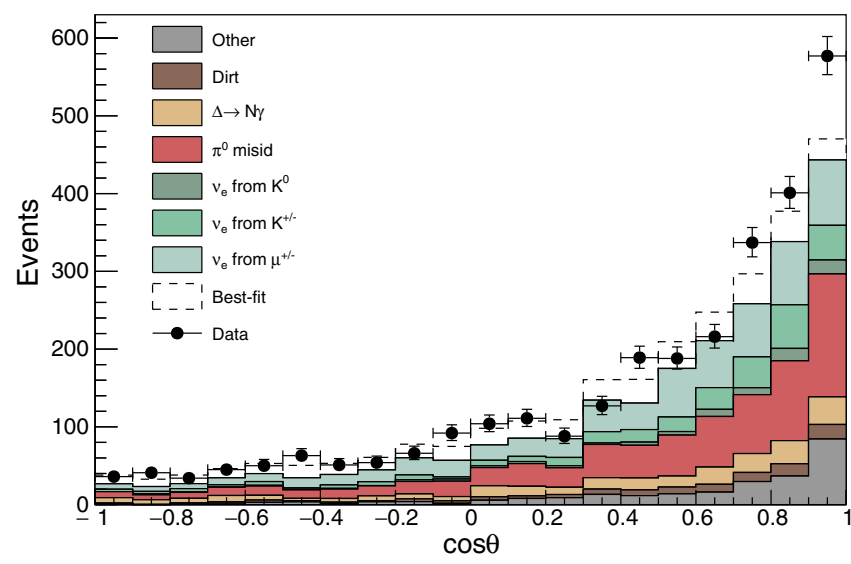

FIG. 8. The MiniBooNE neutrino mode $\cos \theta$ distributions, corresponding to the total $18.75 \times 10^{20}$ POT data in the $200<$ $E_{\nu}^{\mathrm{QE}}<1250 \mathrm{MeV}$ energy range, for $\nu_{e} \mathrm{CCQE}$ data (points with statistical errors) and background (colored histogram). The dashed histogram shows the best fit to the neutrino-mode data assuming two-neutrino oscillations.

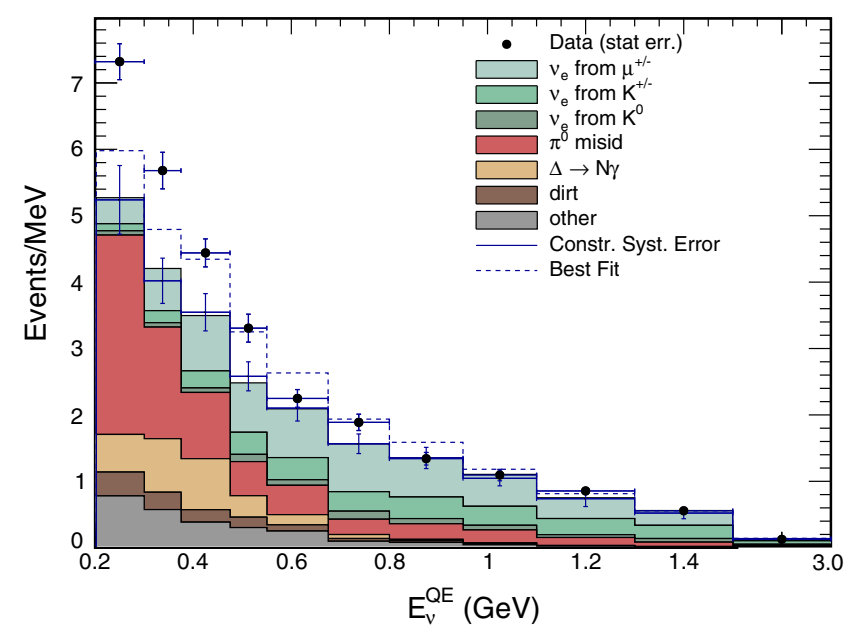

FIG. 9. The MiniBooNE neutrino mode $E_{\nu}^{\mathrm{QE}}$ distributions, corresponding to the total $18.75 \times 10^{20}$ POT data in the $200<$ $E_{\nu}^{\mathrm{QE}}<3000 \mathrm{MeV}$ energy range, for $\nu_{e} \mathrm{CCQE}$ data (points with statistical errors) and predicted backgrounds (colored histograms). The constrained background is shown as additional points with systematic error bars. The dashed histogram shows the best fit to the neutrino-mode data assuming two-neutrino oscillations. The last bin is for the energy interval from 1500-3000 MeV.

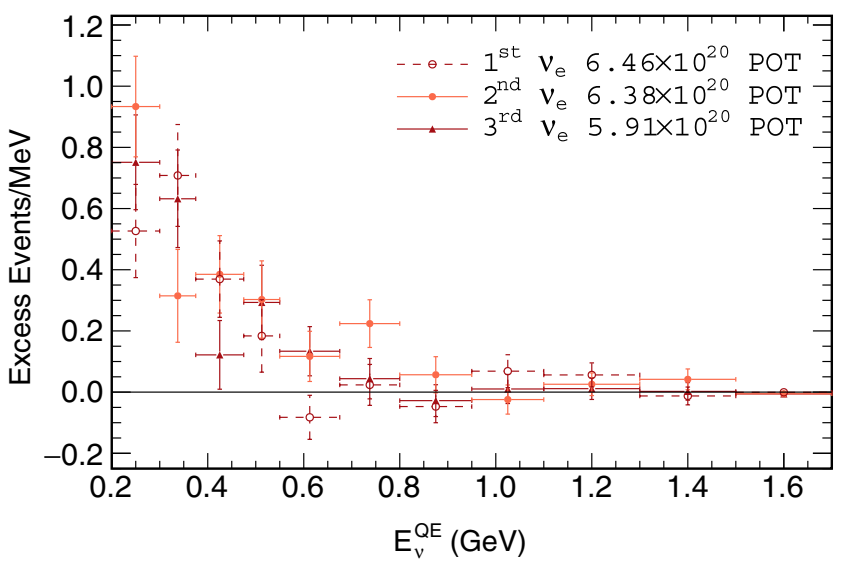

FIG. 10. The total event excesses in neutrino mode as a function of $E_{\nu}^{\mathrm{QE}}$ for the first, second, and third running periods. Error bars include only statistical uncertainties.

distribution of "true" generated neutrino energies, which can overlap adjacent bins. In neutrino mode, a total of 2870 data events pass the $\nu_{e} \mathrm{CCQE}$ event selection requirements with $200<E_{\nu}^{\mathrm{QE}}<1250 \mathrm{MeV}$, compared to a background expectation of $2309.4 \pm 48.1$ (stat.) \pm 109.5 (syst.) events. The excess, as shown in Table II, is then $560.6 \pm 119.6$ events or a $4.7 \sigma$ effect. Figure 10 shows the event excesses as a function of $E_{\nu}^{\mathrm{QE}}$ in neutrino mode for the first, second, and third running periods. Combining the MiniBooNE neutrino and antineutrino data [3], there are a total of 3348 events in the $200<E_{\nu}^{\mathrm{QE}}<1250 \mathrm{MeV}$ energy region, compared to a background expectation of $2710.0 \pm$ 52.1 (stat.) \pm 122.2 (syst.) events. This corresponds to a total $\nu_{e}$ plus $\bar{\nu}_{e} \mathrm{CCQE}$ excess of $638.0 \pm 52.1$ (stat.) \pm 122.2(syst.) events with respect to expectation, where the statistical uncertainty is the square root of the background estimate and the systematic uncertainty includes correlated and uncorrelated systematic parameters. The overall significance of the excess, $4.8 \sigma$, is limited by systematic uncertainties, assumed to be Gaussian, as the statistical significance of the excess is $12.2 \sigma$. The fractional unconstrained systematic uncertainties in the $200<E_{\nu}^{\mathrm{QE}}<$ $1250 \mathrm{MeV}$ energy range are shown in Table III.

In order to test physics models, the numbers of data events, unconstrained background events, and excess events in neutrino mode with visible energy between

TABLE II. The number of data events, background events, and excess events in neutrino mode for different selection criteria. The errors include both statistical and systematic uncertainties. Also shown is the significance of each event excess. $\mathrm{R}$ is the radius of the reconstructed event interaction point.

\begin{tabular}{|c|c|c|c|c|}
\hline Selection & Data & Background & Excess & Significance \\
\hline $200<E_{\nu}^{\mathrm{QE}}<1250 \mathrm{MeV}$ and $R<5 \mathrm{~m}$ & 2870 & $2309.4 \pm 119.6$ & $560.6 \pm 119.6$ & $4.7 \sigma$ \\
\hline $150<E_{\nu}^{\mathrm{QE}}<1250 \mathrm{MeV}$ and $R<5 \mathrm{~m}$ & 3172 & $2560.4 \pm 131.5$ & $611.6 \pm 131.5$ & $4.7 \sigma$ \\
\hline $200<E_{\nu}^{\mathrm{QE}}<1250 \mathrm{MeV}$ and $R<4 \mathrm{~m}$ & 1978 & $1519.4 \pm 81.9$ & $458.6 \pm 81.9$ & $5.6 \sigma$ \\
\hline $200<E_{\nu}^{\mathrm{QE}}<1250 \mathrm{MeV}$ and $R<3 \mathrm{~m}$ & 864 & $673.9 \pm 41.2$ & $190.1 \pm 41.2$ & $4.6 \sigma$ \\
\hline
\end{tabular}


TABLE III. The fractional unconstrained systematic uncertainties in the $200<E_{\nu}^{\mathrm{QE}}<1250 \mathrm{MeV}$ energy range.

\begin{tabular}{lc}
\hline \hline Systematic uncertainty & Fraction of event excess \\
\hline Cross section & $35 \%$ \\
Optical model & $23 \%$ \\
$\pi^{+}$production & $14 \%$ \\
Neutrino flux & $7 \%$ \\
$K^{0}$ production & $4 \%$ \\
$K^{+}$production & $4 \%$ \\
\hline \hline
\end{tabular}

150 and $1250 \mathrm{MeV}$ are shown in Figs. 11-13 as functions of visible energy and $\cos \theta$. In these figures, there are 20 columns of visible energy from 150 to $1250 \mathrm{MeV}$ and 20 rows of $\cos \theta$ from -1 to 1 . There are a total of 3182 data

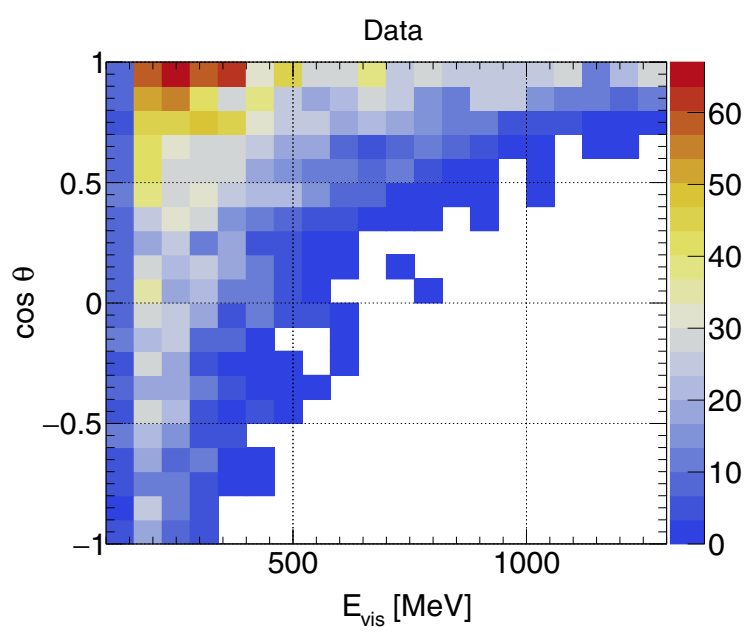

FIG. 11. The total numbers of data events in neutrino mode as functions of reconstructed visible energy and $\cos \theta$. There are 20 columns of visible energy from 150 to $1250 \mathrm{MeV}$ and 20 rows of $\cos \theta$ from -1 to 1 .

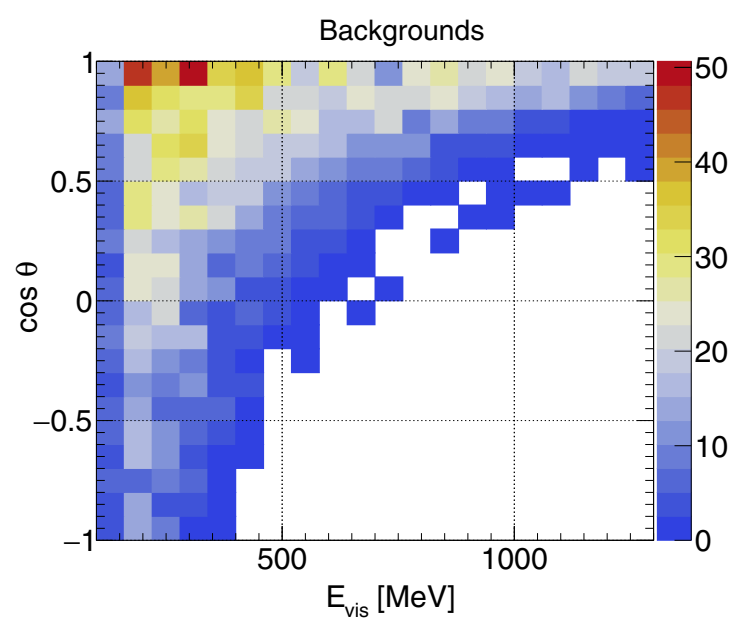

FIG. 12. The total numbers of background events in neutrino mode as functions of reconstructed visible energy and $\cos \theta$. There are 20 columns of visible energy from 150 to $1250 \mathrm{MeV}$ and 20 rows of $\cos \theta$ from -1 to 1 .

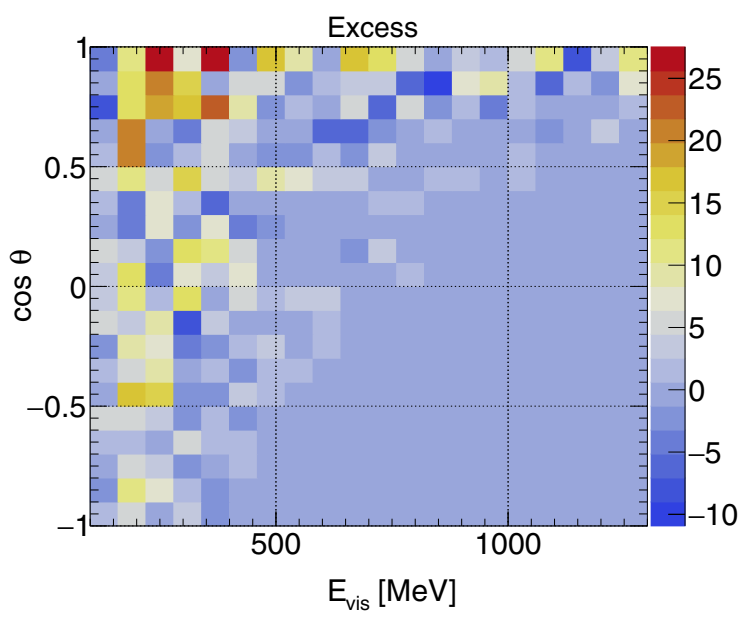

FIG. 13. The total numbers of excess events in neutrino mode as functions of reconstructed visible energy and $\cos \theta$. There are 20 columns of visible energy from 150 to $1250 \mathrm{MeV}$ and 20 rows of $\cos \theta$ from -1 to 1 .

events, 2568.8 background events and 613.2 excess events. Figure 14 shows the $\cos \theta$ distribution of data and background events for the 20 different energy bins, while Fig. 15 shows the $\cos \theta$ distributions from 0.9 to 1 for 10 different visible energy bins. Neutrino-electron elastic scattering events are shown as the hatched region in the "others" category.

Figure 16 shows the number of data and background events as a function of $\cos \theta$ for $\cos \theta>0.9$, where neutrino-electron elastic scattering events are shown as the hatched region in the "others" category and contribute to the $\cos \theta>0.98$ bins. The neutrino-electron elastic events constitute 53\% (89\%) of the "others" category for $\cos \theta>0.90(\cos \theta>0.99)$, and the category also includes neutrino-nucleon charged-current and neutral-current scattering events. As shown in the figure, the excess of data events over background events is approximately the same in each bin.

Figures 17 and 18 show the $E_{\nu}^{\mathrm{QE}}$ and $\cos \theta$ distributions for the $150<E_{\nu}^{\mathrm{QE}}<1250 \mathrm{MeV}$ energy range, and the total event excess as a function of $E_{\nu}^{\mathrm{QE}}$ is shown in Fig. 19. The solid curve on the latter plot shows the two-neutrino oscillation prediction at the best-fit point $\left(\sin ^{2} 2 \theta=0.807\right.$, $\Delta m^{2}=0.043 \mathrm{eV}^{2}$ ). The lowest energy data point has less acceptance than the other data points due to the requirement that the visible energy be greater than $140 \mathrm{MeV}$. Table II lists the number of data events, background events, excess events, and excess significance for the $150<E_{\nu}^{\mathrm{QE}}<1250 \mathrm{MeV}$ energy range.

\section{NEUTRINO OSCILLATION FITS}

Figure 20 shows the MiniBooNE allowed regions in both neutrino mode and antineutrino mode [3] for events with $200<E_{\nu}^{\mathrm{QE}}<3000 \mathrm{MeV}$ within a two-neutrino oscillation 


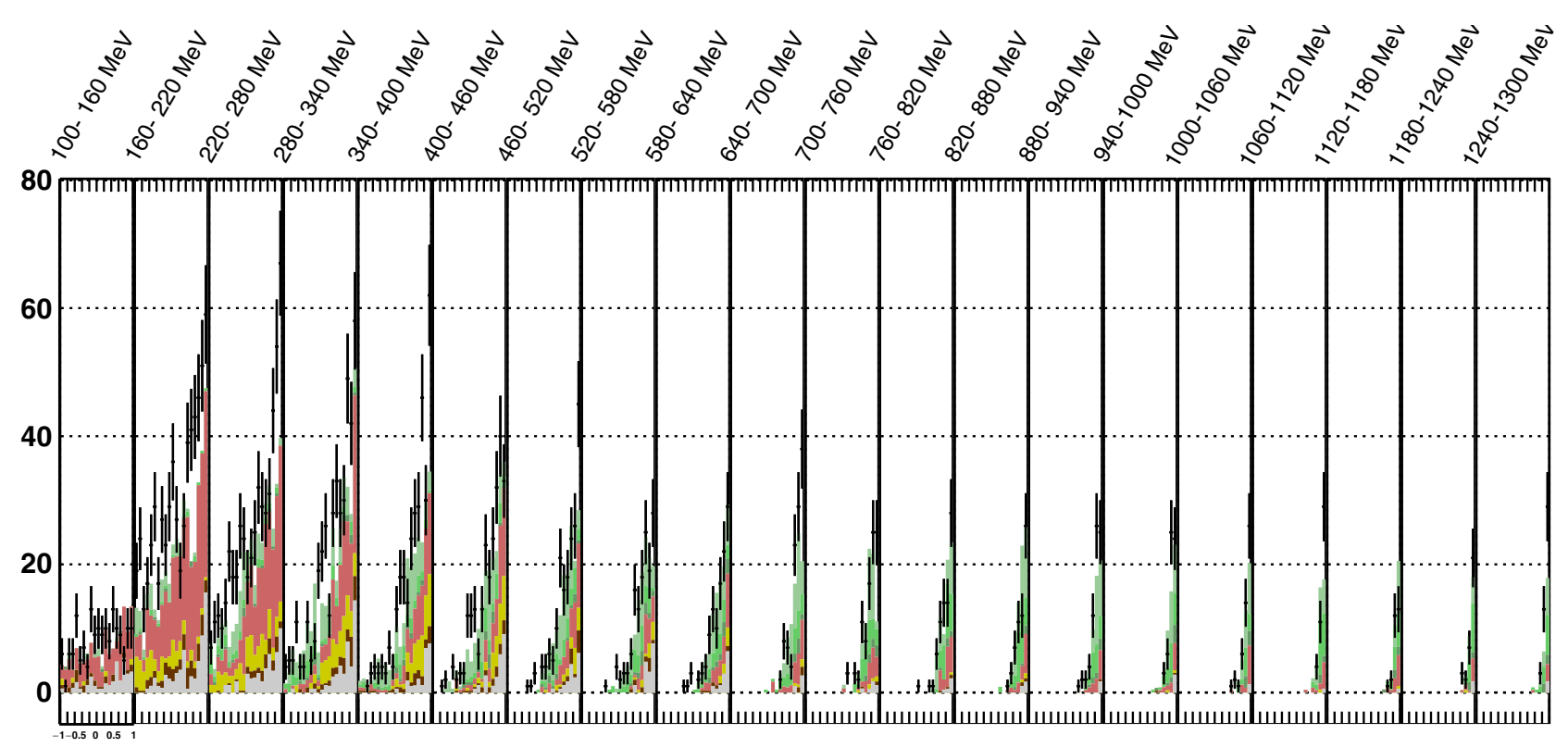

Data compared to stacked backgrounds

$\cos \theta$ for slices of $E_{\text {vis }}[\mathrm{MeV}]$

FIG. 14. The $\cos \theta$ distribution of data events (points with error bars) and background events (colored histogram) in neutrino mode for the 20 different visible energy bins from 150 to $1250 \mathrm{MeV}$.

model. For this oscillation fit the entire data set is used and includes the $18.75 \times 10^{20}$ POT data in neutrino mode and the $11.27 \times 10^{20}$ POT data in antineutrino mode. Also shown are $90 \%$ C.L. limits from the KARMEN [27] and
OPERA [28] experiments. The best combined neutrino oscillation fit occurs at $\left(\sin ^{2} 2 \theta, \Delta m^{2}\right)=\left(0.807,0.043 \mathrm{eV}^{2}\right)$. The $\chi^{2} / n d f$ for the best-fit point in the energy range $200<E_{\nu}^{\mathrm{QE}}<1250 \mathrm{MeV}$ is $21.7 / 15.5$ with a probability of

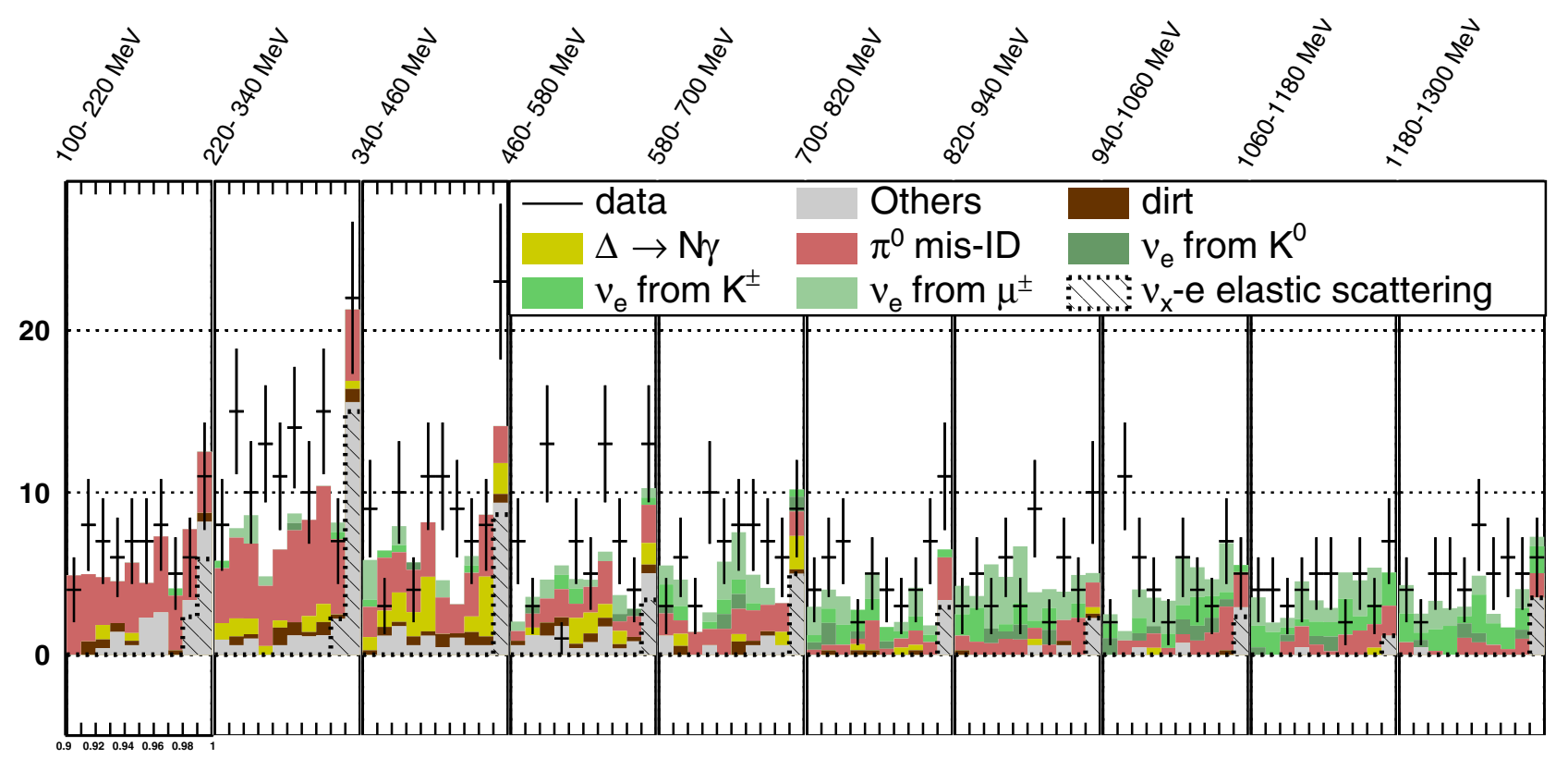

\section{Data compared to stacked backgrounds \\ $\cos \theta$ for slices of $E_{\text {vis }}[\mathrm{MeV}]$}

FIG. 15. The $\cos \theta$ distributions from 0.9 to 1 of data events (points with statistical errors) and background events (histogram) in neutrino mode for ten different visible energy bins from 150 to $1250 \mathrm{MeV}$. Neutrino-electron elastic scattering events are shown as the hatched region in the "others" category. 


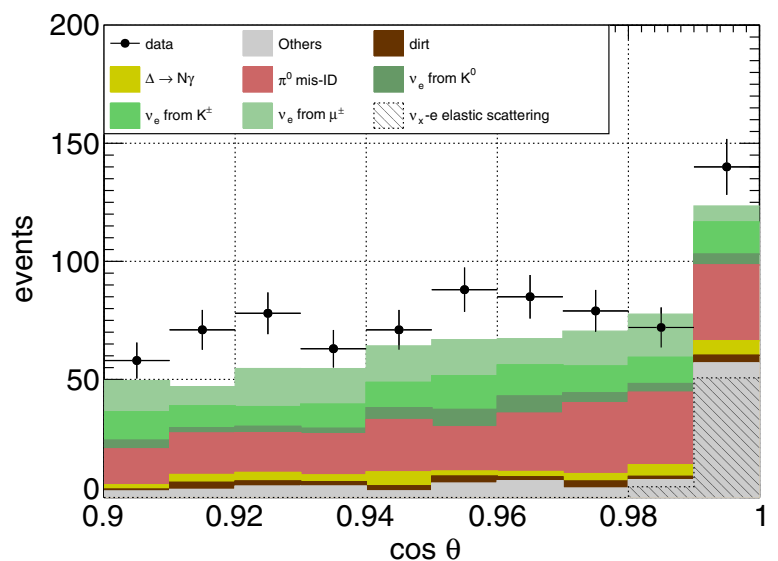

FIG. 16. The MiniBooNE neutrino mode $\cos \theta$ distribution for $\cos \theta>0.9$, corresponding to the total $18.75 \times 10^{20}$ POT neutrino data in the visible energy range from 150 to $1250 \mathrm{MeV}$, for $\nu_{e}$ CCQE data (points with statistical errors) and predicted backgrounds (colored histograms). Neutrino-electron elastic scattering events are shown as the hatched region in the "others" category.

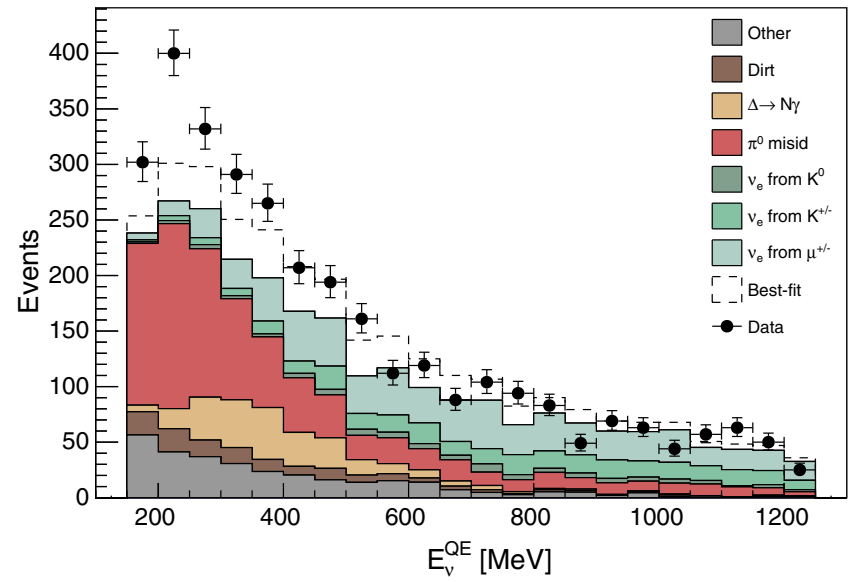

FIG. 17. The MiniBooNE neutrino mode $E_{\nu}^{\mathrm{QE}}$ distributions, corresponding to the total $18.75 \times 10^{20}$ POT neutrino data in the $150<E_{\nu}^{\mathrm{QE}}<1250 \mathrm{MeV}$ energy range, for $\nu_{e} \mathrm{CCQE}$ data (points with statistical errors) and predicted backgrounds (colored histograms). The dashed histogram shows the best fit to the neutrinomode data assuming two-neutrino oscillations.

$12.3 \%$, and the background-only fit has a $\chi^{2}$ probability of $3 \times 10^{-7}$ relative to the best oscillation fit and a $\chi^{2} / n d f=$ $50.7 / 17.3$ with a probability of $0.01 \%$.

Figure 21 compares the $L / E_{\nu}^{\mathrm{QE}}$ distributions for the MiniBooNE data excesses in neutrino mode and antineutrino mode to the $L / E$ distribution from LSND [1]. The error bars show statistical uncertainties only. As shown in the figure, there is agreement among all three data sets. Assuming two-neutrino oscillations, the curves show fits to the MiniBooNE data described above. The significance of

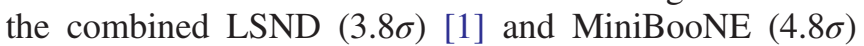
excesses is $6.1 \sigma$, which is obtained by adding the

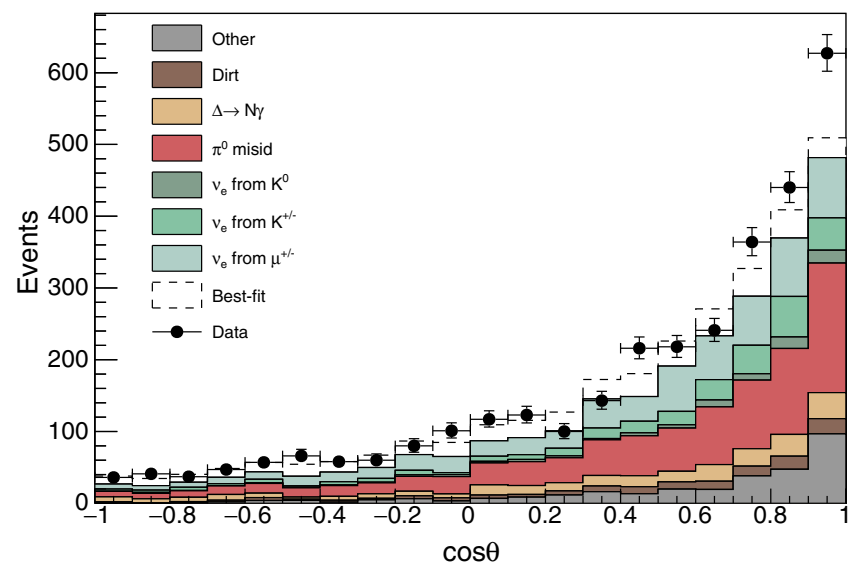

FIG. 18. The MiniBooNE neutrino mode $\cos \theta$ distributions, corresponding to the total $18.75 \times 10^{20}$ POT neutrino data in the $150<E_{\nu}^{\mathrm{QE}}<1250 \mathrm{MeV}$ energy range, for $\nu_{e} \mathrm{CCQE}$ data (points with statistical errors) and predicted backgrounds (colored histograms). The dashed histogram shows the best fit to the neutrinomode data assuming two-neutrino oscillations.

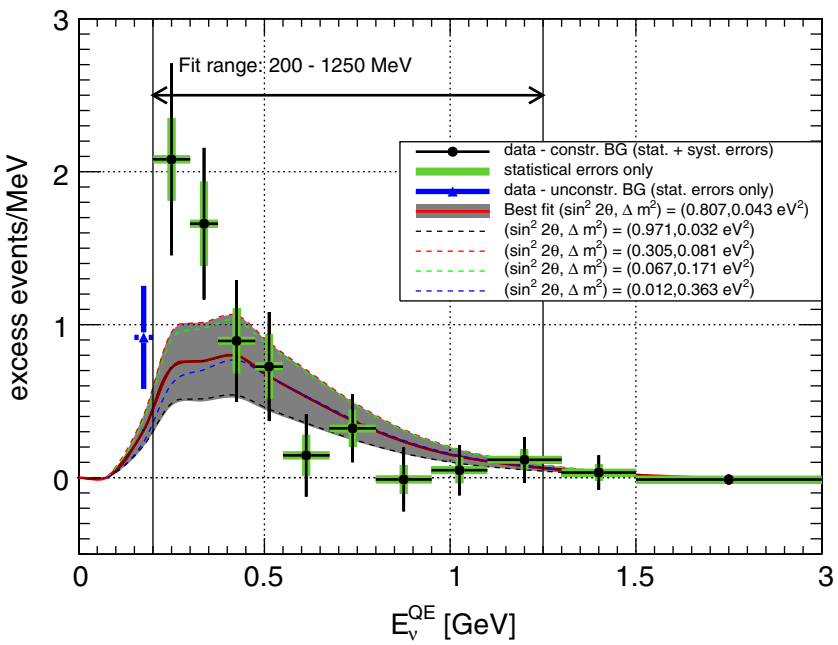

FIG. 19. The total event excess in neutrino mode, corresponding to $18.75 \times 10^{20}$ POT in the $150<E_{\nu}^{\mathrm{QE}}<3000 \mathrm{MeV}$ energy range. The solid curve shows the best fit to the neutrino-mode and antineutrino-mode data in the $200<E_{\nu}^{\mathrm{QE}}<1250 \mathrm{MeV}$ energy range assuming two-neutrino oscillations, while the dashed curves show selected points on the 1 sigma contour. Also shown is the 1-sigma allowed band. The outer error bars include statistical plus constrained systematic uncertainties, while the inner error bars show the statistical uncertainties. The lowest energy data point shows only the unconstrained background statistical uncertainty.

significances in quadrature, as the two experiments have completely different neutrino energies, neutrino fluxes, reconstructions, backgrounds, and systematic uncertainties.

\section{BACKGROUND STUDIES AND CONSTRAINTS}

Constraints have been placed on the various backgrounds in Table I by direct measurements of these 


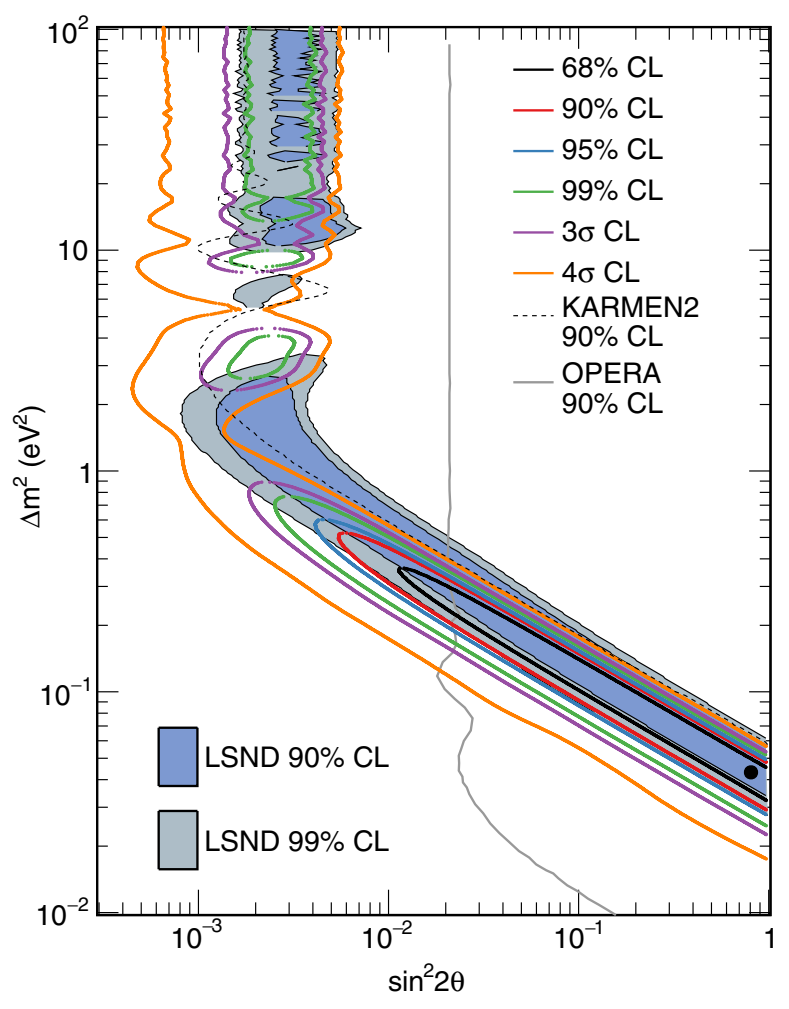

FIG. 20. MiniBooNE allowed regions for combined neutrino mode $\left(18.75 \times 10^{20}\right.$ POT $)$ and antineutrino mode $\left(11.27 \times 10^{20}\right.$ POT) data sets for events with $200<E_{\nu}^{\mathrm{QE}}<3000 \mathrm{MeV}$ within a two-neutrino oscillation model. The shaded areas show the $90 \%$ and $99 \%$ C.L. LSND $\bar{\nu}_{\mu} \rightarrow \bar{\nu}_{e}$ allowed regions. The black point shows the MiniBooNE best fit point. Also shown are $90 \%$ C.L. limits from the KARMEN [27] and OPERA [28] experiments.

backgrounds in the MiniBooNE detector. The $\nu_{\mu} \mathrm{CC}$ background has been well measured [29] by using the Michel electrons from muon decay to identify the event topology. Likewise, the NC $\pi^{0}$ background has also been well measured [30] by reconstructing the two-gamma invariant mass.

In addition, a fit to the vertex radial distribution, shown in Fig. 22, allows a constraint to be placed on the NC $\pi^{0}$ background, due to this background having more events near the edge of the $5 \mathrm{~m}$ radius fiducial volume. ( $\mathrm{NC} \pi^{0}$ events near the edge of the fiducial volume have a greater chance of one photon leaving the detector with the remaining photon then misreconstructing as an electron candidate.) Figure 23 shows the excess event radial distributions, where different processes are normalized to explain the event excess, while Table IV shows the result of log-likelihood shape-only fits to the radial distribution and the multiplicative factor that is required for each hypothesis to explain the observed event excess. The two-neutrino oscillation hypothesis fits the radial distribution best with a $\chi^{2}=8.4 / 9 n d f$, while the NC $\pi^{0}$ hypothesis has a worse fit with a $\chi^{2}=17.2 / 9 n d f$. The intrinsic $\nu_{e}$ backgrounds have a worse $\chi^{2}$ than the two-neutrino

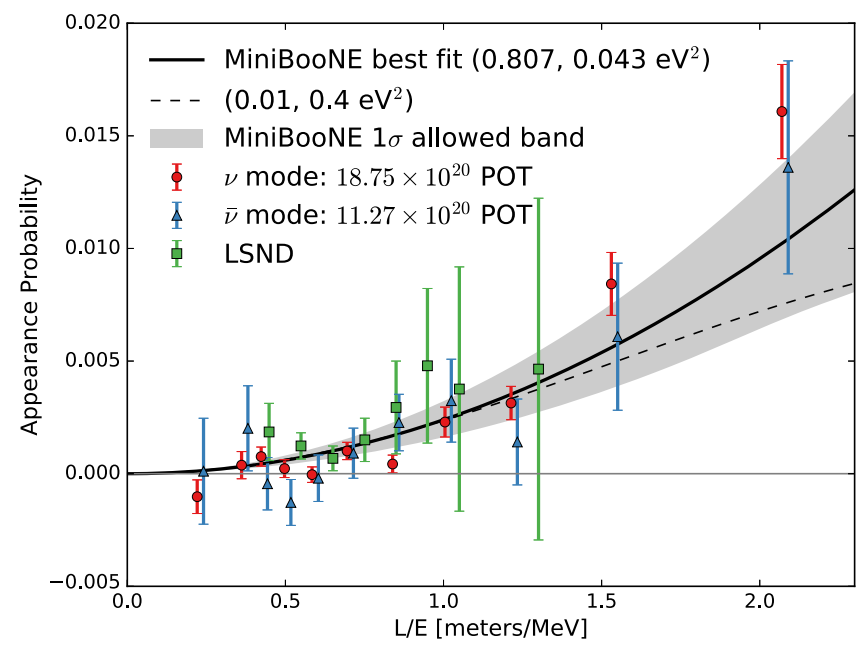

FIG. 21. A comparison between the $L / E_{\nu}^{\mathrm{QE}}$ distributions for the MiniBooNE data excesses in neutrino mode $\left(18.75 \times 10^{20}\right.$ POT) and antineutrino mode $\left(11.27 \times 10^{20}\right.$ POT) to the $L / E$ distribution from LSND [1]. The error bars show statistical uncertainties only. The curves show fits to the MiniBooNE data, assuming two-neutrino oscillations, while the shaded area is the MiniBooNE $1 \sigma$ allowed band. The best-fit curve corresponds to $\left(\sin ^{2} 2 \theta, \Delta m^{2}\right)=\left(0.807,0.043 \mathrm{eV}^{2}\right)$, while the dashed curve corresponds to a $1 \sigma$ fit point at $\left(\sin ^{2} 2 \theta, \Delta m^{2}\right)=\left(0.01,0.4 \mathrm{eV}^{2}\right)$.

oscillation hypothesis due to higher energy $\nu_{e}$ events having a different radial distribution than lower energy $\nu_{e}$ events.

Single-gamma backgrounds from external neutrino interactions ("dirt" backgrounds) are estimated using topological and spatial cuts to isolate the events whose vertices are near the edge of the detector and point towards the detector center [31]. The external event background estimate has been confirmed by measuring the absolute time of signal

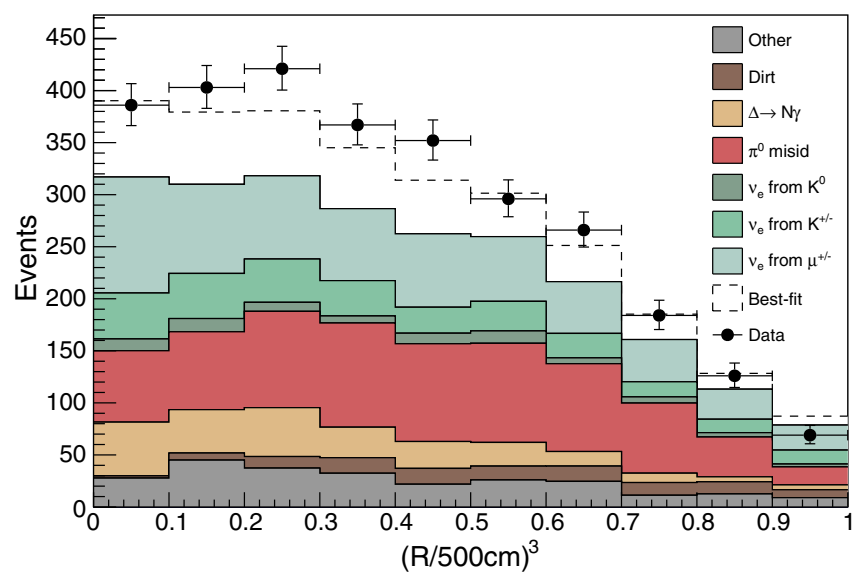

FIG. 22. The MiniBooNE radial vertex distribution, corresponding to the total $18.75 \times 10^{20}$ POT data in neutrino mode in the $200<E_{\nu}^{\mathrm{QE}}<1250 \mathrm{MeV}$ energy range, for $\nu_{e} \mathrm{CCQE}$ data (points with statistical errors) and background (histogram). The dashed histogram shows the best fit to the neutrino-mode data assuming two-neutrino oscillations. 


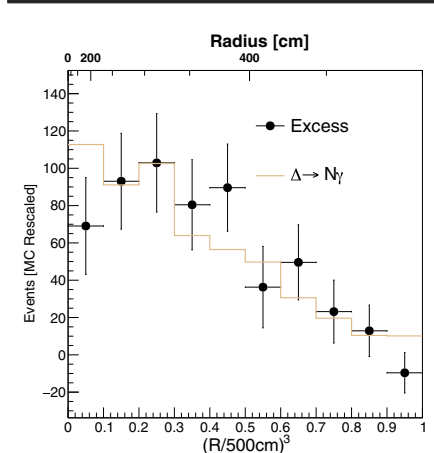

(a)

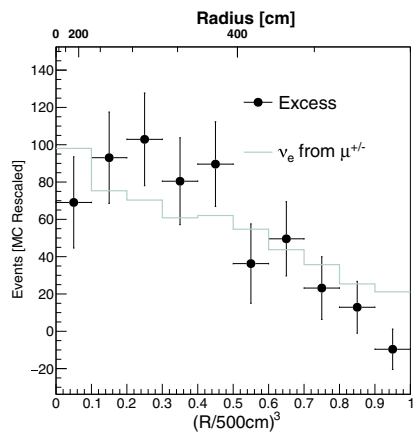

(e)

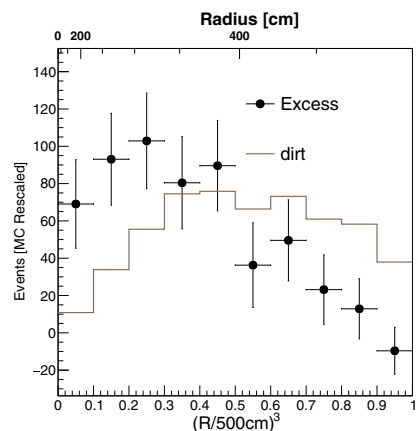

(b)

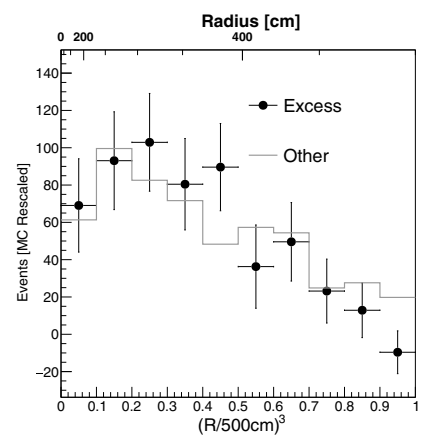

(f)

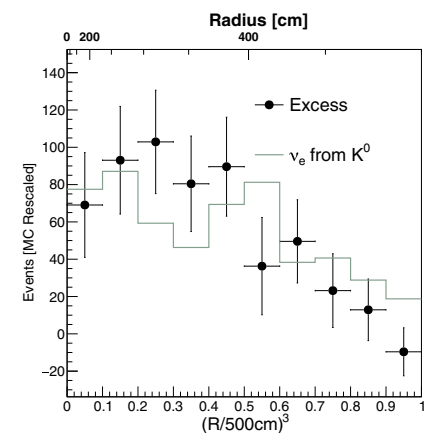

(c)

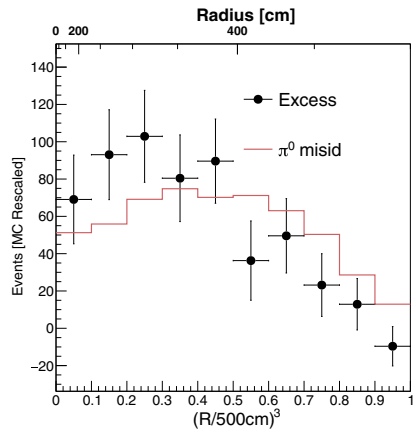

(g)

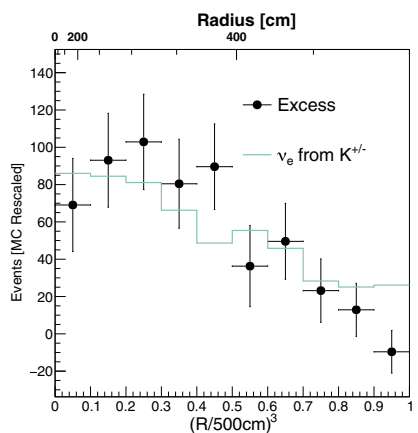

(d)

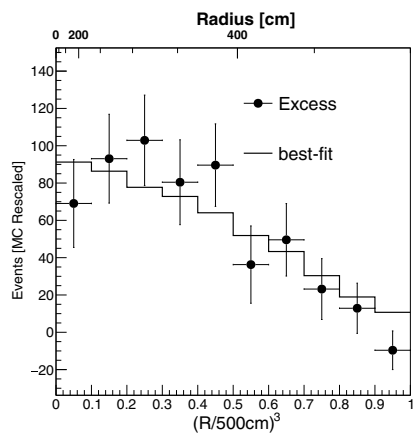

(h)

FIG. 23. The excess event radial distributions in neutrino mode with only statistical errors in the $200<E_{\nu}^{\mathrm{QE}}<1250 \mathrm{MeV}$ energy range, where different processes are normalized to explain the event excess. The different processes are the following: (a) $\Delta \rightarrow N \gamma$; (b) external events; (c) $\nu_{e}$ and $\bar{\nu}_{e}$ from $K_{L}^{0}$ decay; (d) $\nu_{e}$ and $\bar{\nu}_{e}$ from $K^{ \pm}$decay; (e) $\nu_{e}$ and $\bar{\nu}_{e}$ from $\mu^{ \pm}$decay; (f) other $\nu_{e}$ and $\bar{\nu}_{e}$; (g) NC $\pi^{0}$; (h) best fit oscillations.

events relative to the proton beam microstructure (52.81 MHz extraction frequency), which corresponds to buckets of beam approximately every 18.9 ns. Figure 24 shows that the event excess peaks in the $8 \mathrm{~ns}$ window associated with beam bunch time, as expected from

TABLE IV. The result of log-likelihood shape-only fits to the radial distribution in neutrino mode, assuming only statistical errors, where different processes are normalized to explain the observed event excess. The two-neutrino hypothesis fits the radial distribution best with a $\chi^{2}=8.4 / 9 n d f$, while the NC $\pi^{0}$ hypothesis has a worse fit with a $\chi^{2}=17.2 / 9 n d f$. Also shown is the multiplicative factor that is required for each hypothesis to explain the observed event excess.

\begin{tabular}{lcc}
\hline \hline & $\begin{array}{c}\text { Multiplicative } \\
\text { factor }\end{array}$ & $\chi^{2} / 9 n d f$ \\
\hline Nypothesis $\Delta \rightarrow N \gamma$ background & 3.18 & 10.0 \\
External event background & 5.98 & 44.9 \\
$\nu_{e}$ and $\bar{\nu}_{e}$ from $K_{L}^{0}$ decay & 7.85 & 14.8 \\
$\quad$ background & & \\
$\nu_{e}$ and $\bar{\nu}_{e}$ from $K^{ \pm}$decay & 2.95 & 16.3 \\
$\quad$ background & & \\
$\nu_{e}$ and $\bar{\nu}_{e}$ from $\mu^{ \pm}$decay background & 1.88 & 16.1 \\
Other $\nu_{e}$ and $\bar{\nu}_{e}$ background & 3.21 & 12.5 \\
NC $\pi^{0}$ background & 1.75 & 17.2 \\
Best fit oscillations & 1.24 & 8.4 \\
\hline \hline
\end{tabular}

neutrino events in the detector, and is inconsistent with external neutrino events or beam-off events, which would be approximately flat in time. Also, the observed background level outside of the beam agrees well with the predicted background estimate. In addition, good agreement is obtained for the event excess with $\cos \theta>0.9$. The timing reconstruction performed here is similar to the reconstruction in Ref. [24], but with a different time offset applied.

The $\Delta \rightarrow N+\gamma$ background is determined from the NC $\pi^{0}$ event sample [30], which has contributions from $\Delta$ production in ${ }^{12} \mathrm{C}(52.2 \%), \Delta$ production in $\mathrm{H}_{2}(15.1 \%)$, coherent scattering on ${ }^{12} \mathrm{C}(12.5 \%)$, coherent scattering on $\mathrm{H}_{2}(3.1 \%)$, higher-mass resonances (12.9\%), and nonresonant background (4.2\%). The fraction of $\Delta$ decays to $\pi^{0}$ is $2 / 3$ from the Clebsch-Gordon coefficients, and the probability of pion escape from the ${ }^{12} \mathrm{C}$ nucleus is estimated to be $62.5 \%$. The $\Delta$ radiative branching fraction is $0.60 \%$ for ${ }^{12} \mathrm{C}$ and $0.68 \%$ for $\mathrm{H}_{2}$ after integration over all the invariant mass range, where the single gamma production branching ratio increases below the pion production threshold. With these values, the ratio of single gamma events to $\mathrm{NC} \pi^{0}$ events, $R$, can be estimated to be

$$
\begin{aligned}
R & =0.151 \times 0.0068 \times 1.5+0.522 \times 0.0060 \times 1.5 / 0.625 \\
& =0.0091 .
\end{aligned}
$$




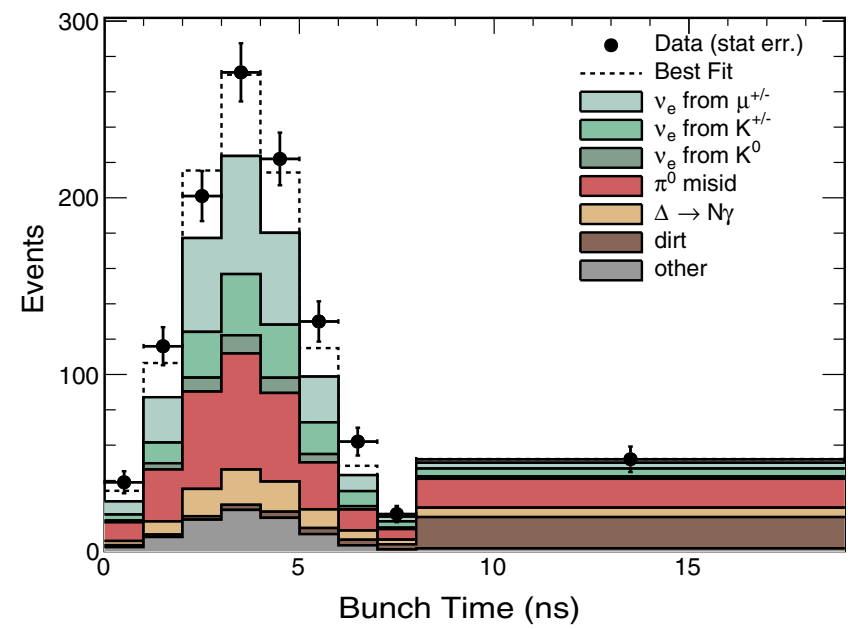

FIG. 24. The bunch timing for data events in neutrino mode compared to the expected background in the $200<E_{\nu}^{\mathrm{QE}}<$ $1250 \mathrm{MeV}$ energy range. Almost all of the excess data events occur, as expected from neutrino events in the detector, within the first $8 \mathrm{~ns}$ of the bunch timing. This data sample uses events collected with the new fiber timing system and represents about $40 \%$ of the entire neutrino mode sample.

Note that single gamma events are assumed to come entirely from $\Delta$ radiative decay. The total uncertainty on this ratio is $14.0 \%(15.6 \%)$ in neutrino (antineutrino) mode. This estimate of $R=0.0091 \pm 0.0013$ agrees fairly well with theoretical calculations of the single gamma event rate [26].

The intrinsic $\nu_{e}$ background comes almost entirely from muon and kaon decay-in-flight in the beam decay pipe. MiniBooNE $\nu_{\mu}$ CCQE event measurements [29] constrain the size and energy dependence of the intrinsic $\nu_{e}$ background from muon decay, while the intrinsic $\nu_{e}$ background from kaon decay is constrained by fits to kaon production data and SciBooNE measurements [32]. Furthermore, due to the higher energy of the intrinsic $\nu_{e}$ background, this background is disfavored from the fit to the radial distribution, as shown in Table IV.

Finally, backgrounds from exotic $\pi^{0}$ decay in the neutrino production target are ruled out from the MiniBooNE beam-dump run, where the incident proton beam was steered above the Be target and interacted in the steel beam dump at the downstream end of the decay pipe. No excess of events was observed [24], which set limits on light dark matter and other exotic $\pi^{0}$ decays.

\section{CONSTRAINTS ON NC $\pi^{0}$ BACKGROUND WITH TIGHTER RADIUS SELECTION}

Explanations for the event excess have included unsimulated photons entering the detector from external interactions and the undersimulation of photons lost from $\pi^{0}$ production within the detector. To test these explanations in a model-independent way, we can use our higher event

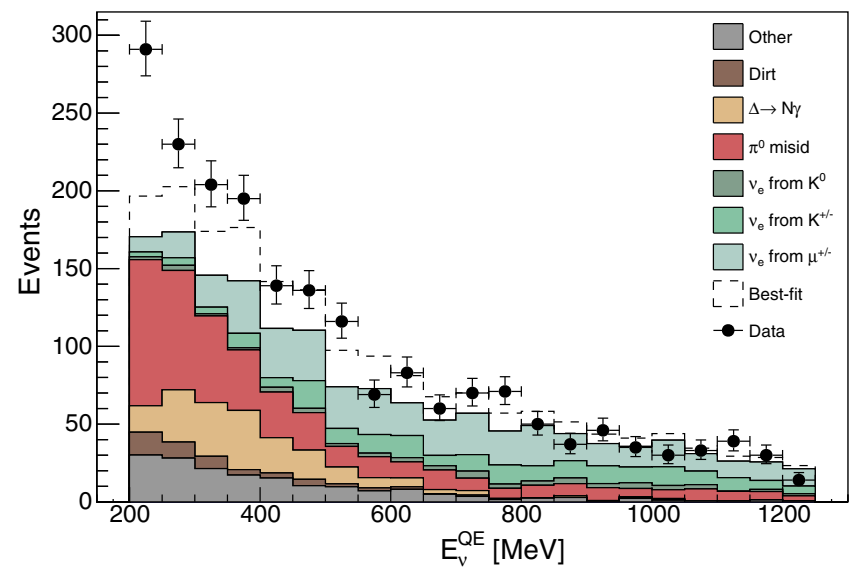

FIG. 25. The MiniBooNE neutrino mode $E_{\nu}^{\mathrm{QE}}$ distributions, corresponding to the total $18.75 \times 10^{20}$ POT data in neutrino mode in the $200<E_{\nu}^{\mathrm{QE}}<1250 \mathrm{MeV}$ energy range, for $\nu_{e} \mathrm{CCQE}$ data (points with statistical errors) and background (histogram) with radius less than $4 \mathrm{~m}$. The dashed histogram shows the best fit to the neutrino-mode data assuming two-neutrino oscillations.

statistics to study the change in the excess as a function of tighter fiducial volume cuts. The $\mathrm{NC} \pi^{0}$ and external event backgrounds preferentially populate higher radius compared to electron neutrino interactions. Therefore, reducing the fiducial radius is expected to reduce the significance of the excess if it is due to these backgrounds and increase the significance of the excess if its distribution is $\nu_{e}$-like. If we change the standard $5 \mathrm{~m}$ cut to $4 \mathrm{~m}$, we find there are 1978 data events in neutrino mode, $1519.4 \pm 81.9$ background events, and an excess of $458.6 \pm 81.9$ events $(5.6 \sigma)$. If we use a $3 \mathrm{~m}$ cut, we find 864 data events, $673.9 \pm 41.2$ background events, and an excess of $190.1 \pm 41.2$ events $(4.6 \sigma)$, consistent with what is expected if the signal is more

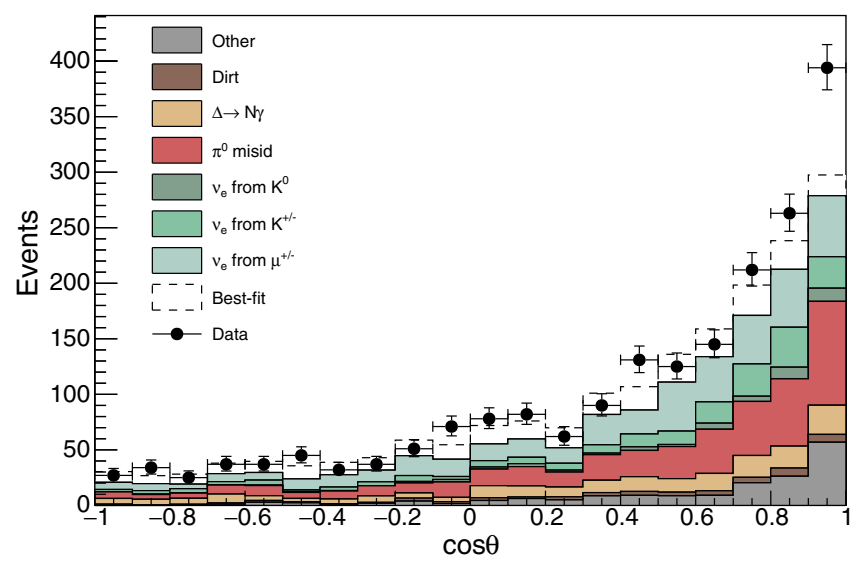

FIG. 26. The MiniBooNE neutrino mode $\cos \theta$ distributions, corresponding to the total $18.75 \times 10^{20}$ POT data in neutrino mode in the $200<E_{\nu}^{\mathrm{QE}}<1250 \mathrm{MeV}$ energy range, for $\nu_{e} \mathrm{CCQE}$ data (points with statistical errors) and background (histogram) with radius less than $4 \mathrm{~m}$. The dashed histogram shows the best fit to the neutrino-mode data assuming two-neutrino oscillations. 
$\nu_{e}$-like. The event statistics are shown in Table II, while Figs. 25 and 26 show the reconstructed neutrino energy and $\cos \theta$ distributions for electron-like events with radius less than $4 \mathrm{~m}$ for both data events and background events.

\section{CONCLUSION}

In summary, the MiniBooNE experiment observes a total excess of $638.0 \pm 52.1$ (stat.) \pm 132.8 (syst.) electronlike events in the energy range $200<E_{\nu}^{\mathrm{QE}}<1250 \mathrm{MeV}$ in both neutrino and antineutrino running modes. The overall significance of the excess, $4.8 \sigma$, is limited by systematic uncertainties, assumed to be Gaussian, as the statistical significance of the excess is $12.2 \sigma$. All of the major backgrounds are constrained by in situ event measurements. Beam timing information shows that almost all of the excess is in time with neutrinos that interact in the detector. The radius distribution shows that the excess is distributed throughout the volume, while tighter cuts on the fiducal volume increase the significance of the excess. The data likelihood ratio disfavors models that explain the event excess due to entering or exiting photons. The MiniBooNE event excess will be further studied by the Fermilab shortbaseline neutrino (SBN) program [33] and by the $\mathrm{JSNS}^{2}$ experiment at J-PARC [34].

\section{ACKNOWLEDGMENTS}

We acknowledge the support of Fermilab, the Department of Energy, and the National Science Foundation, and we acknowledge Los Alamos National Laboratory for LDRD funding.
[1] C. Athanassopoulos et al., Phys. Rev. Lett. 75, 2650 (1995); 77, 3082 (1996); 81, 1774 (1998); Phys. Rev. C. 54, 2685 (1996); 58, 2489 (1998); A. Aguilar et al., Phys. Rev. D 64, 112007 (2001).

[2] A. A. Aguilar-Arevalo et al., Phys. Rev. Lett. 98, 231801 (2007); 102, 101802 (2009); 105, 181801 (2010); 110, 161801 (2013).

[3] A. A. Aguilar-Arevalo et al., Phys. Rev. Lett. 121, 221801 (2018).

[4] M. Sorel, J. M. Conrad, and M. H. Shaevitz, Phys. Rev. D 70, 073004 (2004).

[5] G. Karagiorgi, Z. Djurcic, J. M. Conrad, M. H. Shaevitz, and M. Sorel, Phys. Rev. D 80, 073001 (2009); 81, 039902(E) (2010).

[6] G. H. Collin, C. A. Argüelles, J. M. Conrad, and M. H. Shaevitz, Phys. Rev. Lett. 117, 221801 (2016).

[7] C. Giunti and M. Laveder, Phys. Lett. B 706, 200 (2011); Phys. Rev. D 84, 073008 (2011).

[8] S. Gariazzo, C. Giunti, M. Laveder, and Y. F. Li, J. High Energy Phys. 06 (2017) 135.

[9] S. Boser, C. Buck, C. Giunti, J. Lesgourgues, L. Ludhova, S. Mertens, A. Schukraft, and M. Wurm, Prog. Part. Nucl. Phys. 111, 103736 (2020).

[10] J. Kopp, M. Maltoni, and T. Schwetz, Phys. Rev. Lett. 107, 091801 (2011); J. Kopp, P. A. N. Machado, M. Maltoni, and T. Schwetz, J. High Energy Phys. 05 (2013) 050.

[11] M. Dentler, A. Hernandez-Cabezudo, J. Kopp, P. Machado, M. Maltoni, I. Martinez-Soler, and T. Schwetz, J. High Energy Phys. 08 (2018) 010.

[12] K. N. Abazajian et al., arXiv:1204.5379.

[13] J. M. Conrad, C. M. Ignarra, G. Karagiorgi, M. H. Shaevitz, and J. Spitz, Adv. High Energy Phys. 2013, 1 (2013).

[14] A. Diaz, C. A. Argüelles, G. H. Collin, J. M. Conrad, and M. H. Shaevitz, Phys. Rep. 884, 1 (2020).

[15] J. Asaadi, E. Church, R. Guenette, B. J. P. Jones, and A. M. Szelc, Phys. Rev. D 97, 075021 (2018); G. Karagiorgi, M.H. Shaevitz, and J.M. Conrad, arXiv:1202.1024;
H. Paes, S. Pakvasa, and T. J. Weiler, Phys. Rev. D 72, 095017 (2005); D. Doring, H. Pas, P. Sicking, and T. J. Weiler, Eur. Phys. J. C 80, 1202 (2020).

[16] V. A. Kostelecky and M. Mewes, Phys. Rev. D 69, 016005 (2004); T. Katori, V. A. Kostelecky, and R. Tayloe, Phys. Rev. D 74, 105009 (2006); J. S. Diaz and V. A. Kostelecky, Phys. Lett. B 700, 25 (2011); Phys. Rev. D 85, 016013 (2012).

[17] S. N. Gninenko, Phys. Rev. Lett. 103, 241802 (2009); S. N. Gninenko and D. S. Gorbunov, Phys. Rev. D 81, 075013 (2010); Y. Bai, R. Lu, S. Lu, J. Salvado, and B. A. Stefanek, Phys. Rev. D 93, 073004 (2016); Z. Moss, M. H. Moulai, C. A. Argüelles, and J. M. Conrad, Phys. Rev. D 97, 055017 (2018); E. Bertuzzo, S. Jana, P. A. N. Machado, and R. Z. Funchal, Phys. Rev. Lett. 121, 241801 (2018); P. Ballett, S. Pascoli, and M. Ross-Lonergan, Phys. Rev. D 99, 071701 (2019); O. Fischer, A. Hernandez-Cabezudo, and T. Schwetz, Phys. Rev. D 101, 075045 (2020); M. H. Moulai, C. A. Argüelles, G. H. Collin, J. M. Conrad, A. Diaz, and M. H. Shaevitz, Phys. Rev. D 101, 055020 (2020); M. Dentler, I. Esteban, J. Kopp, and P. Machado, Phys. Rev. D 101, 115013 (2020); A. de Gouvea, O. L. G. Peres, S. Prakash, and G. V. Stenico, J. High Energy Phys. 07 (2020) 141; C. A. Argüelles, M. Hostert, and Y.-D. Tsai, Phys. Rev. Lett. 123, 261801 (2019); A. Datta, S. Kamali, and D. Marfatia, Phys. Lett. B 807, 135579 (2020); A. Abdullahi, M. Hostert, and S. Pascoli, arXiv:2007.11813.

[18] B. Dutta, S. Ghosh, and T. Li, Phys. Rev. D 102, 055017 (2020); W. Abdallah, R. Gandhi, and S. Roy, J. High Energy Phys. 12 (2020) 188; W. Abdallah, R. Gandhi, and S. Roy, arXiv:2010.06159.

[19] J. Liao and D. Marfatia, Phys. Rev. Lett. 117, 071802 (2016).

[20] M. Carena, Y.-Y. Li, C. S. Machado, P. A. N. Machado, and C. E. M. Wagner, Phys. Rev. D 96, 095014 (2017).

[21] A. A. Aguilar-Arevalo et al., Nucl. Instrum. Methods Phys. Res., Sect. A 599, 28 (2009). 
[22] R. B. Patterson, E. M. Laird, Y. Liu, P. D. Meyers, I. Stancu, and H. A. Tanaka, Nucl. Instrum. Methods Phys. Res., Sect. A 608, 206 (2009).

[23] A. A. Aguilar-Arevalo et al., Phys. Rev. D 81, 092005 (2010); 88, 032001 (2013).

[24] A. A. Aguilar-Arevalo et al. (MiniBooNE Collaboration), Phys. Rev. Lett. 118, 221803 (2017); Phys. Rev. D 98, 112004 (2018).

[25] A. A. Aguilar-Arevalo et al., Phys. Rev. D 84, 072005 (2011).

[26] D. Rein and L. M. Sehgal, Phys. Lett. 104B, 394 (1981); S. S. Gershtein, Yu. Ya. Komachenko, and M. Yu. Khlopov, Sov. J. Nucl. Phys. 33, 860 (1981); J. A. Harvey, C. T. Hill, and R. J. Hill, Phys. Rev. Lett. 99, 261601 (2007); T. Leitner, O. Buss, U. Mosel, and L. Alvarez-Ruso, Proc. Sci., NUFACT08 (2008) 009 [arXiv:0809.3986]; J. P. Jenkins and T. Goldman, Phys. Rev. D 80, 053005 (2009); A. M. Ankowski, O. Benhar, T. Mori, R. Yamaguchi, and M. Sakuda, Phys. Rev. Lett. 108, 052505 (2012); K. M. Graczyk, D. Kielczewska, P. Przewlocki, and J. T. Sobczyk, Phys. Rev. D 80, 093001 (2009); V. P. Efrosinin,
Yu. G. Kudenko, and A. N. Khotjantsev, Phys. At. Nucl. 72, 459 (2009); R. J. Hill, Phys. Rev. D 81, 013008 (2010); R. J. Hill, Phys. Rev. D 84, 017501 (2011); X. Zhang and B. D. Serot, Phys. Lett. B 719, 409 (2013); Phys. Rev. C 86, 035502 (2012); 86, 035504 (2012); B. D. Serot and X. Zhang, Phys. Rev. C 86, 015501 (2012); E. Wang, L. Alvarez-Ruso, and J. Nieves, Phys. Rev. C 89, 015503 (2014); Phys. Lett. B 740, 16 (2015).

[27] B. Armbruster et al., Phys. Rev. D 65, 112001 (2002).

[28] N. Agafonova et al., J. High Energy Phys. 06 (2018) 151.

[29] A. A. Aguilar-Arevalo et al., Phys. Rev. D 81, 092005 (2010); Phys. Rev. Lett. 100, 032301 (2008).

[30] A. A. Aguilar-Arevalo et al., Phys. Rev. D 81, 013005 (2010); Phys. Lett. B 664, 41 (2008).

[31] A. A. Aguilar-Arevalo et al., Phys. Rev. Lett. 102, 101802 (2009).

[32] G. Cheng et al., Phys. Rev. D 84, 012009 (2011); C. Mariani, G. Cheng, J. M. Conrad, and M. H. Shaevitz, Phys. Rev. D 84, 114021 (2011).

[33] M. Antonello et al., arXiv:1503.01520.

[34] M. Harada et al., arXiv:1310.1437. 